\title{
Ungarische Ophthalmologische Gesellschaft Budapest
}

Vorsitzender: Prof. J. v. Imre sen.

Bericht vom Sekretär Dr. Gustav Horay.

Sitzung vom 30. Oktober ig2!

Demonstrationen:

A.v.Fazakas: 'Tuberkulose der Sklera. Der tuberkulöse Ursprung der Geschwulst konnte erst durch die histologische Untersuchung er-wiesen werden. Vortragender sah bei Sklera-tbc. gute Erfolge nach Röntgenbestrahlung. Die charakteristische Lage der Tuberkel in der Sklera kann damit erklärt werden, daß die ziliaren Gefäße an dieser Stelle die Sklera durchbohren.

R. Huber: Färbung' der Hornhaut mit Goldchlorid. Die von Knapp vorgeschlagene Methode wurde bei drei Kranken erprobt: bei zwei Leukomen war der Eingriff erfolglos, im dritten Falle wurde ein ge-sunder Teil der Hornhaut vor einem Kolobom mit vorzüglichem Erfolge gefärbt. Reizlosigkeit, gleichmäßige und dauerhafte Färbung. Bei derben Vernarbungen kann das Goldchlorid nicht in die tieferen Schichten eindringen. Deshalb bleibe man bei Leukomen bei den bis-herigen Verfahren.

Aussprache: L. v. Blaskovics; Das Goldchlorid verspricht gute Erfolge beim Färben zu optischem Zweck.

3. G. v. Fodor: Fall von einseitiger Degeneratio pigmentosa retinae.Rechtes Auge normal, am linken typische Entartungen am Fundus.Hemeralopie, eingeengtes Gesichtsfeld. Lues.

4. A. Kreiker: Zwei kleine Modiîikatíonen in der Ausführung derSchattenprobe. Die eine Modifikation besteht aus einem $1 \mathrm{~m}$ langenschwarzen Band mit zwei Schlingen an den Enden. Das Band wirdmit Hilfe der in die Schlingen eingezogenen Gazebinden an die Stirnedes Untersuchten und des Arztes angebracht. Es soil die Distanz von $1 \mathrm{~m}$ bei der Schattenprobe sichern. Dabei kann aber der Arzt auchnäher zum Patienten rücken. Vortragender sah die Vorteile dieses $\lambda \wedge$ erfahrens besonders bei der Zylinder-Skiaskopie.

Die andere Modifikation ist folgende: Urn die Zylinderachse bei der Zylinderskiaskopie sichtbar zu machen, bringt Vortragender eine dünne, schwarze Linie auf die Gläser, die der Achse entspricht, und zwar mit Tusche oder mit einer geschwärzten Einritzung. Damit erzielte er, daß man sich nicht immer die bisher unsichtbare Achse des in der Probebrille gedrehten Zylinderglases zu merken braucht. Die Achse des Zylinders wird vor der Pupille sichtbar; damit ist es auch leichter zu " kontrollieren, ob sich das Lichtband parallel der Zylinderachse bewegt oder nicht.

Kreiker hofft, daß besonders die zweite kleine Änderung zur wei-teren und breiteren Anwendung der vollkommensten objektiven Re-íraktionsbestimmung: der Zylinderskiaskopie, beitragen wird.

Klinische Mitteilungen. 1. I. v. Csapody: Röntgenauînahmen der Arterien in der Augcnum§ebung. Vortragender hat die A. maxillaris externa und die A. transUngarische Ophthalmologische Gesellschaft Budapest. 
versa faciei am Leichenkopf mit Lipiodol injiziert und dadurch sehr gute Röntgenaufnahmen bekommen, die auch feinere Gefäße zum Vor-schein bringen. Das Verfahren ermöglicht ein genaues und schnelles Aufarbeiten eines großen Materials, womit die plastischen Operationen neue Grundlagen bekommen, indem man sich bei der Auswahl der Operationsmethode auf die genaueren Kenntnisse der Gefäßlehre des Gesichtes stötzen wird.

2. St. v. Tapasztó: Drei Fäüe von Tenonitis. Von diesen waren zweiim Zusammenhange mit Influenza und Rheumatismus, der dritte miteinem Hornhautgeschwüre Heilung.

Aussprache: J. v. Imre sen.: Erwähnt zwei Fälle, die er vor vielen Jahren veröffentlicht hat. Bemerkenswert ist jener, wo die Tenonitis im Bilde einer Panophthalmitis verlief. Auch hier Heilung. Er halt bei diesen Erkrankungen ias Atophan für besser, als die Salizylprä-parate. 3. Z. v. Ferenczy: Über Refraktionsstörung bei Grlaukom. Bei einemKranken mit Glaucoma simplex entwickelte sich binnen zwei Jahreneine Myopie von 5 Dioptrien. Langsame Progression der Myopie.Tension mit Pilocarpin ständig normal. Vortragender sucht den Zusammenhang zwischen Glaukom und Myopie. Es könnte als Ursacheder Refraktionsstörung die Erweiterung des hinteren Bulbusabschnittesund die Anderung des Refraktionsindex der Linse angenommen werden.

Aussprache: R. Huber: Erwähnt einen ähnlichen Fall, bei dem bei noch normaler Tension auf einmal 4 D Myopie entstancl. Hornhaut, Linse, Fundus gaben keine Erklärung dazu. Nach dem Erscheinen dieser Refraktionsstörung stieg die Tension auf $82 \mathrm{~mm}$ : eine subkon-junktivale Injektion von $0.15 \mathrm{~g}$ Suprareninlösung hat den Druck wieder normalisiert. Die Refraktion wurde auch wieder normal, wie vorher. Sehschärfe: 5/6.

Vorträge.

J.v.Aszalôs: Die Erkcnnungswerte der verschicdenen Sehproben. Auf der Augenklinik in Debrecen wurden Untersuchungen mit den Zeichen der Blaskovics-, Heß-, Liebermann- und Snellenschen Sehproben vorgenommen. Auf ihren Erkennungswert wurden 46 mehr oder weniger verschiedene Zeichen geprüft (auch die zwei modifizierten Landolt-Ringe Kreikers). Die Versuche wurden mit Zeichen von 8,7 mm Größe an-gestellt, deren kleinste Teile in einem Winkel von 1' aus 30 m Ent-fernung erkennbar waren. Im allgemeinen betrug die Sehschärfe 90 Oxyoptrien (:i·5). Die am leichtesten erkennbaren Zeichen waren: L und A, der schwierigste Buchstabe: B. Der Wertunterschied in der Er-kennung zwischen L und B war $43 \% \bullet$ Die Differenz der schwierigsten und leichtesten Zeichen war bei Sehproben nach Blaskovics: 20\%, Snellen: 43\%, Csapody: 4i\%, Liebermann: 43\%, Heß: 11\%. Die kleinste Anderung eines Zeichens kann den Wert desselben im sehr großen Maße $(6-8 \%)$ verändern.

L. v. Blaskovics zeig't seine neuen Oxyoptrie-Tíîieln. Die zweite Auflage erschien vor kurzem in ungarischer und englischer Sprache. Es wurde eine außerordentliche Genauigkeit in der typographischen Her-stellung der Tafeln dadurch erreicht, daß er die Vervielfältigung auf Ungaris · che Ophthalmologische Gesellschaft Budapest.

photographischer resp. zinkographischer Weise durchgeführt hat. In der zweiten Auflage wurden an zwei Zeichen (2 und 7) kleine Ände-rungen vorgenommen.

Aussprache: A. Kreiker: Seine Modifikation am Landolt-Zeichen will das Prinzip der Sehschärfe mit strenger Genauigkeit zustande-bringen, was eigentlich nur mit Hilfe eines kreis- oder scheibenförmigen Zeichens zu erreichen ware. K. nahm selbst als Untersuchter bei den Versuchen von Aszalôs teil und hatte wiederholt Gelegenheit, sich über die große Bedeutung der relativen Erkennung zu überzeugen. Bloß ein Stimmungswechsel kann eine 40-50\% $/ 0$ ige Sehschärfenänderung ver-ursachen. 
3. R. Huber: Erîahrungen über liintere Sklerotomie bei Gílaskòrper-trübungen. Empfiehlt bei entzündlichen Glaskörpeftrübungen und Blu-tungen statt Absaugung des Glaskörpers (zur Neääen) die einfachere und schonende Sclerotomia posterior. Da das Absaugen mit einer dicken Nadel geschieht, kann der Glaskörper dadurch auch geschädigt werden, was bei der Sklerotomie mit Graefe-Messev nicht geschieht. Er sah gute Erfolge davon.

Aussprache: L. v. Blaskovics: Mit dieser Methode kann man größere Mengen von Glaskörper entleeren als durch Absaugen nach zur Neäden. Keine Schädigung.

B. v. Horváth erwähnt einen Fall von tuberkulöser Iritis, den er mit Quarzlicht und Röntgen erfolglos behandelte, und wo später eine Sklerotomie rasche Besserung brachte.

Diagnose und Therapie.

Meesmann, A. (Aus der Klinik der Charité Berlin), Beiträge zur physi-kalisehen Chemie des intraokularen Flüss $1 / 8$ keitsweehsels unter normalen und pathologischen Verhältnissen. insbesondere beim Cl·laukom. Archiv f. Augenheilkunde, 97. Bd. H. 1. 1925.

Auf Grund der Ergebnisse chemischer Forschungen und klini-scher Feststellungen, die im Original studiert werden müßten, kommt Verf. zu folgenden Haupt-Schlußsätzen: 1. Durch Unterschiede im osmotischen Druck des Blutes ist das Glaukom nicht zu erklären. Die trennenden Membranen zwischen Blut und Kammerwasser sind für Kristalloide vollkommen durchlässig. Unterschiede in der kristalloid-osmotischen Konzentration des Blutes können daher nicht zu länger dauernden Druckveränderungen im inneren Auge führen. Eine solche Möglichkeit besteht nur für den Ao\%o¿áosmotischen Druck. Abwei-chungen vom normalen Kolloiddruck kommen aber im Blut Glaukom-kranker nicht vor (Serr). Auch auf Grund des ,Donnangleichgewichts”1)

1. 1) Das ,,Do $7 / 8$ w $\alpha$ «-Gleichgewicht"

eine Reihe physikalisch-chemischer Gesetzmäßigkeiten

die nach ihrem ersten Untersucher Donnan genannt werden

definiert Verfasser: ,, Werden zwei Lösungen

von denen die eine ein kolloidales Ion enthält

während die andere kolloid/m ist, 